\title{
Crystal Structure of Citrobacter freundii Asp214Ala Tyrosine Phenol-lyase Reveals that Asp214 is Critical for Maintaining a Strain in the Internal Aldimine
}

\author{
Dalibor Milić, ${ }^{\text {a,* }}$ Tatyana V. Demidkina, ${ }^{\mathrm{b}}$ Lyudmila N. Zakomirdina, ${ }^{\mathrm{b}}$ \\ Dubravka Matković-Čalogović, ${ }^{a}$ and Alfred A. Antson ${ }^{\mathrm{c}, *}$ \\ ${ }^{a}$ Department of Chemistry, Faculty of Science, University of Zagreb, Horvatovac 102a, 10000 Zagreb, Croatia \\ ${ }^{\mathrm{b}}$ Engelhardt Institute of Molecular Biology, Russian Academy of Sciences, 32 Vavilov Street, Moscow 119991, Russia \\ 'Structural Biology Laboratory, Department of Chemistry, University of York, Heslington, \\ York YO10 5YW, United Kingdom \\ RECEIVED MAY 11, 2011; REVISED MAY 7, 2012; ACCEPTED MAY 31, 2012

\begin{abstract}
Tyrosine phenol-lyase (TPL) is a pyridoxal-5'-phosphate (PLP) dependent enzyme which catalyzes $\beta$-elimination of L-tyrosine. In the holoenzyme the protonated pyridinium N1 atom of the PLP cofactor is hydrogen-bonded to the side chain of Asp214. Here we report the X-ray structure of $C$. freundii D214A TPL determined at $1.9 \AA$ resolution. Comparison with the structure of the wild-type TPL shows that the D214A replacement induced significant conformational reorganization in the active site leading to its partial closure. Significantly, in D214A TPL the strain in the internal aldimine is completely released and the pyridine N1 atom of PLP is deprotonated. These observations explain the considerably reduced activity of the D214A TPL towards its substrates [T. V. Demidkina et al., Biochim. Biophys. Acta, Proteins Proteomics 1764 (2006) 1268-1276]. The reported structure reveals that Asp214 is critical for maintaining the strain in the internal aldimine. We argue that this strain is used by the enzyme to accelerate the transaldimination reaction, the first step in the enzymatic catalysis.(doi: 10.5562/cca1915)
\end{abstract}

Keywords: tyrosine phenol-lyase, chemical strain, internal aldimine, pyridoxal 5'-phosphate, X-ray structure

\section{INTRODUCTION}

Pyridoxal-5'-phosphate (PLP)-dependent enzymes are mainly involved in the metabolism of amino acids and biogenic amines. Most of them make the internal aldimine (Schiff base) by covalently binding a cofactor (PLP) molecule to the $\varepsilon$-amino group of a lysine residue in the active site (Figure 1). ${ }^{1}$ Mechanisms of almost all reactions catalyzed by the PLP-dependent enzymes have several common features. The first step of the reaction with an amino acid substrate is transaldimination, i.e. conversion from the internal to external aldimine. It was shown that strain in the internal aldimine, caused by the torsion between the PLP pyridine ring and the imine $\mathrm{C} 4^{\prime}=\mathrm{N} \zeta$ bond (Figure 2), plays a considerable role in the transaldimination reaction catalyzed by aspartate aminotransferase. ${ }^{2,3}$ Similar strain in the internal aldimine was also found in a number of structures of other PLPdependent enzymes. ${ }^{2}$ Moreover, it was demonstrated that exposure to relatively high doses of X-ray radiation relieves the strain in the internal aldimine and even

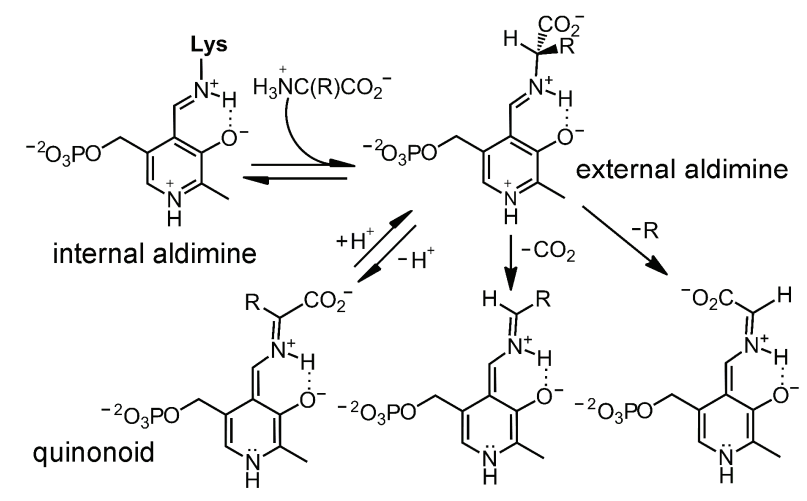

Figure 1. Key intermediates common to almost all PLPdependent enzymes.

induces breakage of the $\mathrm{C} 4^{\prime}=\mathrm{N} \zeta$ bond in phosphoserine aminotransferase. ${ }^{4}$

Heterolytic cleavage of a single bond at the $\mathrm{C} \alpha$ atom of a substrate (Figure 1) is the next step after transaldimination. ${ }^{5}$ In most cases, the role of PLP is

\footnotetext{
$\dagger$ This article belongs to the Special Issue Chemistry of Living Systems devoted to the intersection of chemistry with life.

* Authors to whom correspondence should be addressed. (E-mail: dmilic@chem.pmf.hr; fred.antson@york.ac.uk)
} 

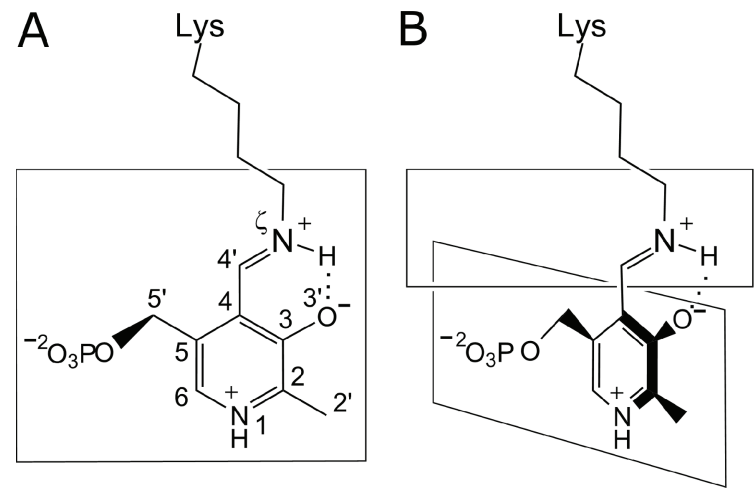

Figure 2. Internal aldimine in the relaxed (A) and the strained (B) state. Definition of the atom names is given for the relaxed structure.

stabilization of the formed carbanionic quinonoid intermediate by delocalization of its negative charge through extensive conjugation of the $\pi$-electrons in the aldimine bond, pyridine ring and phenolate group (deprotonated O3' atom) of PLP. ${ }^{6}$ The protonated form of the pyridine $\mathrm{N} 1$ atom is stabilized by the nearby negatively charged (Asp, Glu) or neutral side chains (Ser, Thr) in a saltbridge and/or hydrogen-bond interaction. In PLPdependent enzymes for which destabilization of the quinonoid intermediate actually enhances the enzymatic reaction, a positively charged residue (e.g. Arg in alanine racemase) ${ }^{7}$ is found in an interaction with the deprotonated $\mathrm{N} 1$ atom.

Tyrosine phenol-lyase (TPL; EC 4.1.99.2) is a PLPdependent enzyme that catalyzes the reversible hydrolytic cleavage ( $\beta$-elimination) of L-tyrosine. It is a homotetrameric protein with the active sites situated at the interface of two subunits that form the catalytic dimer. ${ }^{8-10}$ Previous kinetic studies of Citrobacter freundii TPL mutant enzymes indicated that Asp214 stabilizes PLP in the form with a protonated pyridine nitrogen atom (N1); the data also indicated that Asp214 stabilizes the orientation of the cofactor that is favorable for the enzymatic reaction. ${ }^{11}$ More specifically, D214A and D214N were shown to be inactive for the $\beta$-elimination of L-tyrosine and 3fluoro-L-tyrosine, but they decomposed substrates with better leaving groups at reduced rates (10- to 100 -fold lower). In addition, TPL affinity towards the cofactor PLP was reduced in the mutant enzymes by about four orders of magnitude. In order to rationalize these results, we have determined the structure of $C$. freundii D214A TPL. This structure, presented here, reveals substantial structural reorganization in the active site. In particular, hydrogen bonding interaction between the residue 214 and the N1 atom of the PLP pyridine ring, observed in the wild type protein, is disrupted in the mutant enzyme. Notably, the resulting rotation of the pyridine ring relieves a strain in the conformation of the internal aldimine, explaining the reduced activity of the mutant TPL.

\section{EXPERIMENTAL}

\section{Crystallization}

C. freundii D214A TPL was produced in Escherichia coli SVS370 cells transformed with the plasmid pTZTPLD214A and purified as described before. ${ }^{11}$ Crystals were obtained in crystallization conditions previously established for the wild-type enzyme..$^{12,13}$ Before crystallization, the protein was dialyzed against the solution that contained $0.5 \mathrm{mmol} \mathrm{dm}{ }^{-3}$ PLP and $2 \mathrm{mmol} \mathrm{dm}^{-3}$ dithiothreitol (DTT) in $50 \mathrm{mmol} \mathrm{dm}^{-3}$ triethanolamine (TEA) buffer, $\mathrm{pH}$ 8.0. The protein solution was concentrated to $18-20 \mathrm{mg} \mathrm{cm}^{-3}$ using a $30 \mathrm{~K}$ ultrafiltration membrane concentrator (Filtron). Crystals were grown at $293 \mathrm{~K}$ by the hanging drop vapor diffusion technique by mixing $2 \mu \mathrm{L}$ of the protein solution with an equal volume of the reservoir solution containing $50 \mathrm{mmol} \mathrm{dm}^{-3}$ TEA buffer ( $\mathrm{pH} 8.0$ ), 0.4-0.8 mol $\mathrm{dm}^{-3} \mathrm{KCl}, 0.35-0.38 \mathrm{~g} \mathrm{~cm}^{-3}$ poly(ethylene glycol) 5000 monomethyl ether (PEG $5000 \mathrm{MME}$ ), $5 \mathrm{mmol} \mathrm{dm}^{-3}$ PLP and $2 \mathrm{mmol} \mathrm{dm}{ }^{-3}$ DTT.

\section{Structure Determination and Refinement}

Crystals of D214A TPL were flash-cooled by dipping into liquid nitrogen directly from the crystallization drops, without adding any cryoprotectant. X-ray diffraction data were collected at $100 \mathrm{~K}$, at the BM30A beamline, ESRF (Grenoble), using a wavelength of $0.980 \AA$ and a MAR 165 CCD detector (MarResearch). Data collection and refinement statistics are given in Table 1. The data were processed using DENZO and SCALEPACK. ${ }^{14}$ Since the unit-cell parameters for D214A TPL crystal were only slightly different than for the native TPL holoenzyme, ${ }^{13}$ it was possible to refine the D214A TPL structure in REFMAC ${ }^{15}$ using the wildtype TPL holoenzyme structure (PDB ID: 2EZ1), modified by omitting PLP and solvent atoms, as a starting model. During the refinement, the small (residues 1944, 346-404, 434-456) and the large domain (residues $2-13,45-345,405-422$ ) of each subunit were used as separate TLS ${ }^{16}$ groups. Structural model was built using $\mathrm{COOT}^{17}$ and water molecules were added by ARP/wARP ${ }^{18}$ Other crystallographic calculations were carried out by programs incorporated in the CCP4 software suite. ${ }^{19}$ The final model consists of two crystallographically independent protein subunits forming the catalytic dimer, two $\mathrm{K}^{+}$cations, two covalently bound PLP molecules, 8 PEG fragments, and 748 solvent molecules. All residues, except for the first Nterminal residue of both crystallographically independent protein subunits (Met1), were located in the electron density map. The side chains of residues Met66(A), Ile93(A), Thr124(A), Arg198(A), Lys210(A), Arg381(A), Asp433(A), Lys41(B), Met66(B), Ile93(B), 
Table 1. Crystallographic Data

\begin{tabular}{ll}
\hline Data Collection and Processing & \\
\hline beamline & BM30A, ESRF \\
wavelength / $\AA$ & 0.980 \\
temperature / K & 100 \\
crystal system & orthorhombic \\
space group & $P 2{ }_{1} 2{ }_{1}$ \\
unit-cell parameters & \\
$a / \AA$ & 132.6 \\
$b / \AA$ & 142.7 \\
$c / \AA$ & 59.4 \\
resolution range ${ }^{(a)} / \AA$ & $25.0-1.91(1.97-1.91)$ \\
unique reflections & $87110(8589)$ \\
$R_{\text {merge }} / \%$ & $6.9(49.9)$ \\
average $I / \sigma(I)$ & $15.1(3.1)$ \\
data redundancy & $4.5(4.2)$ \\
completeness $/ \%$ & $99.8(99.4)$ \\
Wilson B-factor $/ \AA^{2}$ & 24.4 \\
\hline
\end{tabular}

Refinement and Model Correlation

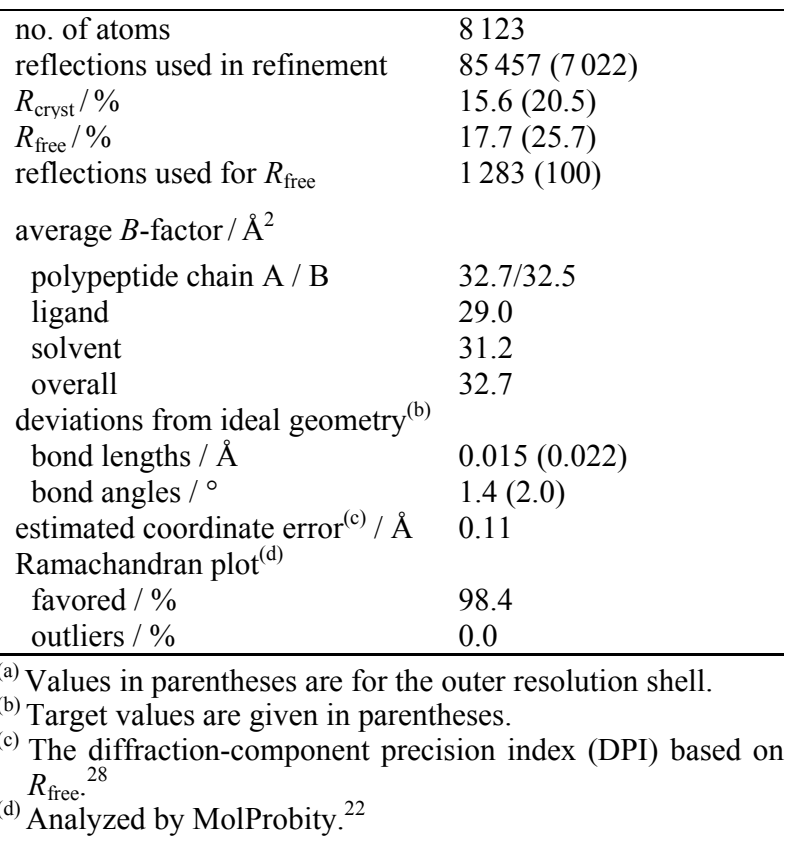

Asn151(B), Arg201(B), Lys210(B), Val283(B), Lys330(B), Ser365(B), and His430(B) were modeled in two conformations with an occupancy of 0.5 . Similarly as in other TPL structures, ${ }^{12,20}$ eight patches of electron density at the protein surface were interpreted as fragments of PEG MME used for crystallization. ${ }^{21}$ The model was validated by MolProbity. ${ }^{22}$ Figures were made in PyMOL. ${ }^{23}$

\section{RESULTS AND DISCUSSION}

\section{Structural Model of D214A TPL}

The crystal structure of the D214A mutant TPL was determined at $1.9 \AA$ resolution. The conformations of the two crystallographically independent subunits (constituting the catalytic dimer) are very similar, with a root mean square deviation (RMSD) of $0.30 \AA$ between their $\mathrm{C} \alpha$ atoms. The largest difference of $1.06 \AA$ is for the $\mathrm{C} \alpha$ atoms of Asn390 which is situated in a flexible loop. Differences between the structure of the D214A TPL and the wild-type TPL ${ }^{13}$ are more pronounced with the $\mathrm{C} \alpha$ atom RMSD of $0.44 \AA$. The largest difference is in the conformation of the small domain (residues 19-44, 346-404, and 434-456; RMSD of $0.77 \AA$ for subunit A, and $0.44 \AA$ for subunit $\mathrm{B}$ ) and a part of the large domain comprising residues 108-211 (RMSD of $0.60 \AA$ and $0.49 \AA$ for subunits $\mathrm{A}$ and $\mathrm{B}$, respectively). RMSDs for other parts of the polypeptide chain are only $0.25 \AA$ and $0.21 \AA$ for subunits $\mathrm{A}$ and $\mathrm{B}$, respectively. There are no significant differences in conformation of the two crystallographically independent active sites of the D214A TPL.

\section{Structural Changes Caused by Asp214 to Ala Substitution}

Replacement of Asp214 by Ala prevents the N1 atom of the PLP's pyridine ring from forming a hydrogenbond/salt-bridge interaction with the side chain of residue 214. This, in turn, changes the geometry of the active site (Figure 3 ) with the pyridine ring being rotated around the $\mathrm{C} 4-\mathrm{C} 4$ ' bond and moved towards Glu103 by $\approx 0.8 \AA$. This is accompanied by a conformational change in the side chain of Lys 257 and formation of the more relaxed internal aldimine geometry which is additionally stabilized by the intramolecular hydrogen bond between the protonated Lys $257 \mathrm{~N} \zeta$ and deprotonated PLP O3' atom. Significantly, in the D214A TPL the torsion angle $\mathrm{C} 3-\mathrm{C} 4-\mathrm{C} 4^{\prime}-\mathrm{N} \zeta$ of the internal aldimine is reduced to $4.2^{\circ}$ (subunit A) or $1.3^{\circ}$ (subunit B), in comparison with $\approx 21^{\circ}$ angle found in the structure of the wild-type holoenzyme. ${ }^{13}$

The most prominent changes in the active site of the D214A TPL are in the conformations of the side chains of Glu103 $\left(\chi_{2}\right.$ is changed by $\left.\approx 120^{\circ}\right)$ and Asn 185 $\left(\chi_{1}\right.$ is changed by $\left.\approx 111^{\circ}\right)$. The side chain of Phe123 is stacked with the pyridine ring of PLP at an angle of $4^{\circ}$ and $7^{\circ}$ in the $\mathrm{A}$ and $\mathrm{B}$ active sites, respectively, which is a somewhat smaller angle than in the wild-type holoenzyme $\left(12^{\circ}\right)$. Helix $\alpha 4^{\prime}$ (residues 124-132), including the active site residues Thr124, Thr125, and Thr126, is moved by $\approx 1.2 \AA$ away from the active site cleft (Figure 4). As a result of this movement, side chains of the three consecutive threonine residues are positioned 1.1-1.5 $\AA$ farther away from the PLP molecule. Despite these changes, due to the conformational adjustments of the small domain and residues 108-211 from the large domain, the active site cleft as a whole is slightly more closed than in the wild-type holoenzyme. 

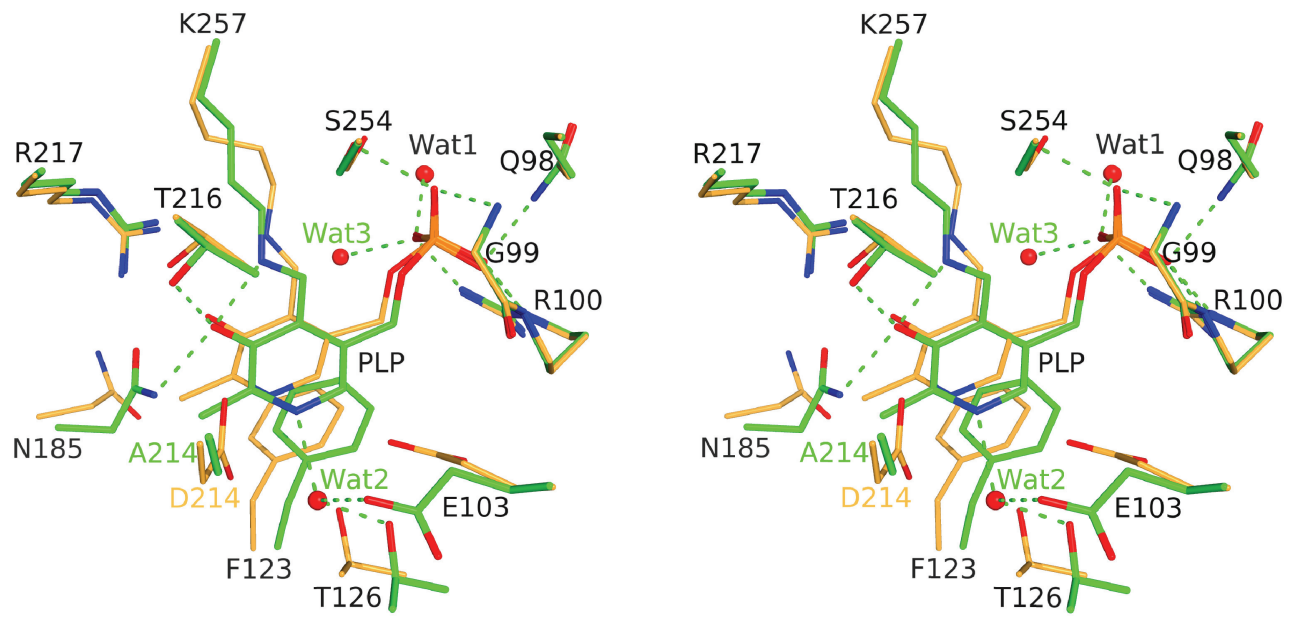

Figure 3. Comparison of the active sites of D214A (green) and wild-type (orange) TPL (a stereo view). Hydrogen bonds in D214A TPL are shown as dashed lines. Solvent molecules Wat2 and Wat3 are only found in the structure of D214A TPL.

New hydrogen bonds, not observed in the structure of the wild-type holoenzyme, are formed between the PLP O3' atom and the side chains of Asn185 and Thr216, as well as between the PLP N1 atom and the nearby solvent molecule, denoted as Wat2 in Figure 3, that is also hydrogen-bonded to the side chains of Glu103 and Thr126. Additionally, a solvent molecule Wat3 makes a hydrogen bond with the phosphate of PLP. Both Wat2 and Wat 3 molecules were not observed in the structure of the wild-type holoenzyme. The hydrogen bond between PLP O3' atom and the side chain

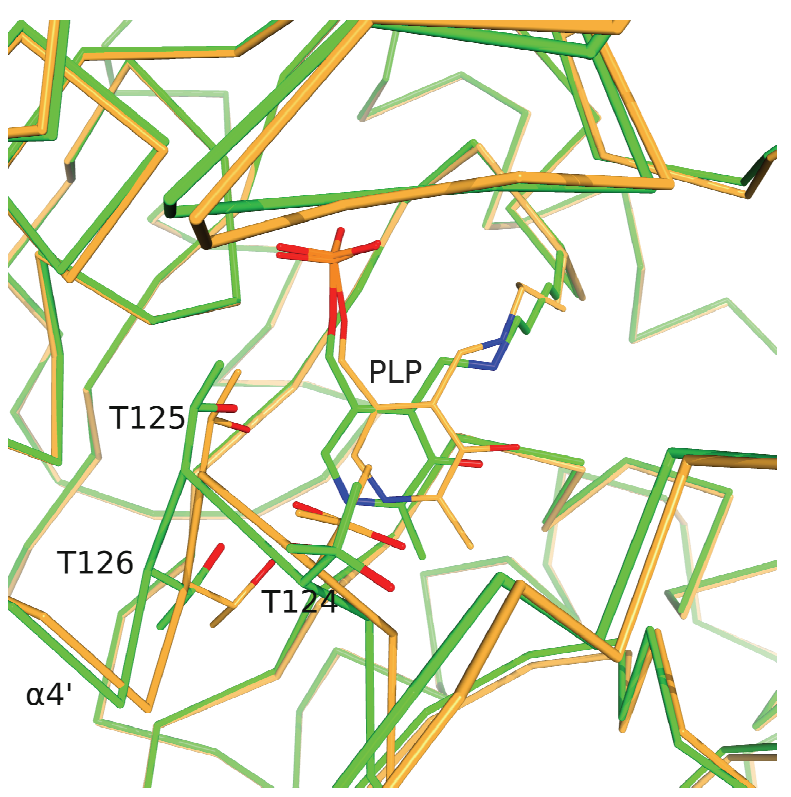

Figure 4. The active-site cleft is slightly more closed in the D214A TPL (green) than in the wild-type TPL (orange). Both structures are shown as $\mathrm{C} \alpha$-traces. of Arg217, observed in the wild-type enzyme, is not present in the structure of D214A TPL.

\section{Functional Implications of the Structural Changes Induced by Asp214 to Ala Substitution}

The loss of the catalytic activity in D214A TPL with Ltyrosine and 3-fluoro-L-tyrosine, as well as considerably reduced activity of this mutant protein with substrates containing good leaving groups, ${ }^{11}$ can be, at least partially, explained by the more relaxed geometry of the internal aldimine. Indeed, the internal aldimine is more stable in the more relaxed state of the D214A active site than in the strained state observed in the wild-type holoenzyme. ${ }^{13}$ The observed stabilization of the groundstate in D214A TPL would decrease the rate of the external aldimine formation.

Spectroscopic observations indicated that the pyridine $\mathrm{N} 1$ atom of PLP is deprotonated. ${ }^{11}$ The deprotonated state is consistent with the network of hydrogen bonding interactions observed in the structure of D214A TPL. The deprotonation at N1 position contributes to the reduced catalytic activity of the mutant enzyme. Namely, the protonated N1 atom of the pyridine ring should additionally stabilize a negative charge generated upon $\mathrm{C} \alpha$ deprotonation and formation of the quinonoid intermediate. Stabilization of the quinonoid intermediate by the protonated (and positively charged) N1 atom of PLP is achieved through the extended system of conjugated $\pi$-electrons (Figure 1). If the $\mathrm{N} 1$ atom is deprotonated, the quinonoid intermediate cannot be stabilized by the described mechanism. In this case the $\mathrm{C} \alpha-\mathrm{H}$ acidity of the external aldimine, as well as the rate of the quinonoid formation, would decrease. 
Similar structural and functional differences between the wild-type and the mutant enzyme were also observed for the structurally related $E$. coli aspartate aminotransferase, ${ }^{24,25}$ in which Asp222 corresponds to the Asp214 in $C$. freundii TPL. The structure of D222A aspartate aminotransferase (PDB entry 1ASB) clearly demonstrates formation of a hydrogen bond between the deprotonated N1 atom of PLP and a nearby solvent molecule, as well as release of strain in the internal aldimine. The same is also noticed for the E177S D-amino acid aminotransferase from Geobacillus stearothermophilus (PDB entry $1 \mathrm{G} 2 \mathrm{~W}$ ). ${ }^{26}$ Namely, in the structure of its wild type, the internal aldimine is found in the strained state with a hydrogenbond/salt-bridge interaction between the protonated N1 atom of PLP and the carboxylate of Glu177. ${ }^{27}$ It appears that residues which directly interact with the N1 atom of the cofactor have dual functional roles: apart from ensuring the productive protonation of N1, they also stabilize the distorted state of the internal aldimine. It is possible that this is a general feature for all PLP-dependent enzymes.

Considerable changes in the active-site conformation are another factor which contributes to the inactivity of D214A TPL towards L-tyrosine and 3-fluoroL-tyrosine. Despite the slightly more closed active-site cleft in D214A TPL compared to the wild type protein, our analysis shows that there is still enough space for the L-tyrosine molecule to enter the active site and to form a Michaelis complex as well as subsequent reaction intermediates. We showed previously that the active-site closure is crucial for the $\beta$-elimination of Ltyrosine and its derivatives. ${ }^{12}$ The closure induces strain in the quinonoid intermediate and thus facilitates elimination of the formally non-activated phenolic moieties. ${ }^{20}$ Additionally, the closure brings catalytically important residues Arg381 and Thr124 into positions suitable for interaction with the substrate. Thr124 in the structure of D214A TPL is moved by $1.0 \AA$ further from PLP. In this conformation the side chain of Thr124 would be too far from the phenolic group of the substrate and thus unable to form a hydrogen bond with its hydroxyl upon closure of the active site. Moreover, the distorted geometry of the active site in D214A TPL could disfavor the active-site closure. On the contrary, this closure is not crucial for the $\beta$-elimination of amino acids with good leaving groups, such as $S$ - $(o-$ nitrophenyl)-L-cysteine, so their $\beta$-elimination reaction is less affected by the conformational reorganization observed in D214A TPL.

Due to the conformational changes in the active site of D214A TPL, there are fewer protein-PLP interactions than in the wild-type holoenzyme. This accounts for the observed difference in Gibbs energies of PLPbinding to D214A TPL and the wild-type apoenzyme $\left(\Delta \Delta_{\mathrm{r}} G=17 \mathrm{~kJ} \mathrm{~mol}^{-1}\right){ }^{11}$

\section{CONCLUSIONS}

Structural data reported here demonstrate that replacement of the Asp214 by Ala in C. freundii TPL induces significant conformational reorganization of the active site. It is evident that Asp214 is not only responsible for the increase in the $\mathrm{p} K_{\mathrm{a}}$ value of the pyridinium nitrogen atom of PLP, but it also has a role in maintaining the strain in the internal aldimine. We argue that the strain is important for accelerating the transaldimination reaction. Asp214 is conserved in many other PLP-dependent enzymes, with structural observations on two other enzymes demonstrating that, like in TPL, this residue stabilizes the distorted state of the internal aldimine. It is possible that this is a general catalytic feature of all PLP-dependent enzymes.

Supplementary Material. - Atomic coordinates and structure factors were deposited with the Protein Data Bank (http://www.pdb.org) as a PDB entry 2YHK.

Acknowledgements. The research was supported by the Ministry of Science, Education and Sports of the Republic of Croatia (Grant 119-1193079-1084 to D.M. and D.M.-Č.), by the Russian Foundation for Basic Research (Grant 11-04-00220-a to T.V.D.), and by the Wellcome Trust (Fellowship 081916 to A.A.A.). We thank the late Dr. Richard Kahn for help during the data collection at beamline BM30A, ESRF, Grenoble.

\section{REFERENCES}

1. A. C. Eliot and J. F. Kirsch, Annu. Rev. Biochem. 73 (2004) 383-415.

2. H. Hayashi, H. Mizuguchi, I. Miyahara, M. M. Islam, H. Ikushiro, Y. Nakajima, K. Hirotsu, and H. Kagamiyama, Biochim. Biophys. Acta, Proteins Proteomics 1647 (2003) 103-109.

3. H. Hayashi, H. Mizuguchi, I. Miyahara, Y. Nakajima, K. Hirotsu, and H. Kagamiyama, J. Biol. Chem. 278 (2003) 9481-9488.

4. A. P. Dubnovitsky, R. B. G. Ravelli, A. N. Popov, and A. C. Papageorgiou, Protein Sci. 14 (2005) 1498-1507.

5. H. C. Dunathan, P. Natl. Acad. Sci. USA 55 (1966) 712-716.

6. J. P. Richard, T. L. Amyes, J. Crugeiras, and A. Rios, Curr. Opin. Chem. Biol. 13 (2009) 475-483.

7. J. P. Shaw, G. A. Petsko, and D. Ringe, Biochemistry 36 (1997) 1329-1342.

8. A. A. Antson, T. V. Demidkina, P. Gollnick, Z. Dauter, R. L. Von Tersch, J. Long, S. N. Berezhnoy, R. S. Phillips, E. H. Harutyunyan, and K. S. Wilson, Biochemistry 32 (1993) 4195-4206.

9. B. Sundararaju, A. A. Antson, R. S. Phillips, T. V. Demidkina, M. V. Barbolina, P. Gollnick, G. G. Dodson, and K. S. Wilson, Biochemistry 36 (1997) 6502-6510.

10. S. V. Pletnev, A. A. Antson, N. I. Sinitsyna, Z. Dauter, M. N. Isupov, E. N. Hurs, N. G. Faleev, K. S. Wilson, G. Dodson, T. V. Demidkina, and E. G. Arutyunyan, Crystallogr. Rep. 42 (1997) 809-819.

11. T. V. Demidkina, N. G. Faleev, A. I. Papisova, N. P. Bazhulina, V. V. Kulikova, P. D. Gollnick, and R. S. Phillips, Biochim. Biophys. Acta, Proteins Proteomics 1764 (2006) 1268-1276. 
12. D. Milić, T. V. Demidkina, N. G. Faleev, D. MatkovićČalogović, and A. A. Antson, J. Biol. Chem. 283 (2008) 29206-29214.

13. D. Milić, D. Matković-Čalogović, T. V. Demidkina, V. V. Kulikova, N. I. Sinitzina, and A. A. Antson, Biochemistry 45 (2006) 7544-7552.

14. Z. Otwinowski and W. Minor, Methods Enzymol. 276 (1997) 307-326.

15. G. N. Murshudov, A. A. Vagin, and E. J. Dodson, Acta Crystallogr., Sect. D 53 (1997) 240-255.

16. M. D. Winn, M. N. Isupov, and G. N. Murshudov, Acta Crystallogr., Sect. D 57 (2001) 122-133.

17. P. Emsley, B. Lohkamp, W. G. Scott, and K. Cowtan, Acta Crystallogr., Sect. D 66 (2010) 486-501.

18. A. Perrakis, R. Morris, and V. S. Lamzin, Nat. Struct. Biol. 6 (1999) 458-463.

19. Collaborative Computational Project, Number 4, Acta Crystallogr., Sect. D 50 (1994) 760-763.

20. D. Milić, T. V. Demidkina, N. G. Faleev, R. S. Phillips, D. Matković-Čalogović, and A. A. Antson, J. Am. Chem. Soc. 133 (2011) 16468-16476.

21. J. Hašek, Z. Kristallogr. Suppl. 23 (2006) 613-618.
22. I. W. Davis, A. Leaver-Fay, V. B. Chen, J. N. Block, G. J. Kapral, X. Wang, L. W. Murray, W. B. Arendall, III, J. Snoeyink, J. S. Richardson, and D. C. Richardson, Nucl. Acids Res. 35 (2007) W375-383.

23. W. L. DeLano, The PyMOL Molecular Graphics System, DeLano Scientific, Palo Alto, CA, USA (http://www.pymol.org), 2002.

24. T. Yano, Y. Hinoue, V. J. Chen, D. E. Metzler, I. Miyahara, K. Hirotsu, and H. Kagamiyama, J. Mol. Biol. 234 (1993) $1218-1229$.

25. J. J. Onuffer and J. F. Kirsch, Protein Eng. 7 (1994) 413-424.

26. P. W. van Ophem, B. W. Lepore, K. Kishimoto, D. Ringe, and J. M. Manning, Studies on an Active Site residue, E177, That Affects Binding of the Coenzyme in D-Amino Acid Transaminase, and Mechanistic Studies on a Suicide Substrate in: A. Iriarte, H. M. Kagan and M. Martinez-Carrion (Ed.), Biochemistry and Molecular Biology of Vitamin B6 and PQQ-dependent Proteins, Birkhäuser Verlag, Basel, Switzerland, 2000, pp. 339-346.

27. D. Peisach, D. M. Chipman, P. W. Van Ophem, J. M. Manning, and D. Ringe, Biochemistry 37 (1998) 4958-4967.

28. D. Cruickshank, Acta Crystallogr., Sect. D 55 (1999) 583-601. 